\title{
La expansión ósea en la implantología oral
}

\author{
VELASCO ORTEGA E* \\ PÉREZ PÉREZ O** \\ PATO MOURELO J*** \\ LORRIO CASTRO JM**** \\ CRUZ VALIÑO JM ***
}

\begin{abstract}
Velasco Ortega E, Pérez Pérez O, Pato Mourelo J, Lorrio Castro JM, Cruz Valiño JM. La expansión ósea en la implantología oral. Av Periodon Implantol. 2008; 20, 2: 95-101.
\end{abstract}

\section{RESUIMEN}

Introducción. El objetivo del presente estudio era mostrar los resultados del tratamiento con implantes dentales insertados mediante la técnica de expansión ósea.

Métodos. 56 pacientes con pérdidas dentales fueron tratados con 117 implantes Microdent ${ }^{\circledR}$ con superficie con chorreado de arena para la rehabilitación mediante la técnica de expansión ósea con los expansores roscados Microdent ${ }^{\circledR}$. Los implantes fueron cargados después de un periodo de cicatrización de 3-6 meses dependiendo de la mandíbula o maxilar superior.

Resultados. Los hallazgos clínicos indican una supervivencia y éxito de los implantes del 98,3\%. 2 implantes se perdieron durante el periodo de cicatrización. El 90,5\% de los implantes fueron insertados en el maxilar superior y el 9,5\% en la mandíbula. Después de un periodo medio de carga funcional de 14,1 meses, no ha habido complicaciones tardías. El 59,1\% de las prótesis realizadas fueron coronas unitarias; el 31,1\% con puentes fijos; el 4,9\% con rehabilitaciones completas fijas y el $4,9 \%$ con sobredentaduras.

Conclusiones. Este estudio indica que el tratamiento con implantes dentales mediante la técnica de expansión ósea constituye una alternativa terapéutica implantológica con éxito.

\section{PALABRAS CLAVE}

Implantes orales, expansión ósea, osteótomos, expansores, implantología oral.

Fecha de recepción: Marzo 2007.

Aceptado para publicación: Abril 2007.

\section{INTRODUCCIÓN}

La implantología oral ha revolucionado la práctica odontológica y ha tenido un rápido desarrollo. Des- pués de la evidencia científica de la posibilidad con éxito de la utilización de los implantes y del conocimiento de los fenómenos biológicos de la oseointegración, la tendencia ha sido hacia la integración sim-

* Profesor Titular de Odontología Integrada de Adultos. Facultad de Odontología. Director del Postgrado de Implantología Oral. Universidad de Sevilla.

** Profesor de Cirugía Oral y MáxiloFacial. Facultad de Estomatología de La Habana (Cuba).

*** Profesor Colaborador Honorario de Odontología Integrada de Adultos. Facultad de Odontología. Universidad de Sevilla.

**** Profesor Responsable de Odontología en Pacientes Especiales. Facultad de Odontología. Universidad Alfonso X El Sabio. Madrid. 
plificada de la técnica quirúrgica y prostodóncica para extenderlas entre la profesión dental ${ }^{1-2}$.

Además, el tratamiento con implantes dentales ha sido mejorado con nuevos protocolos clínicos como la sustitución de la carga diferida o convencional (meses) por precoz (semanas) o inmediata (horas) y la inserción de implantes - con nuevos diseños macro y microscópicos- en localizaciones con pobre calidad o escasa cantidad de hueso ${ }^{1-2}$.

Existen condiciones orales en los pacientes edéntulos totales o parciales como representa el déficit horizontal de la cresta alveolar que dificultan la inserción de implantes lo que obliga al profesional a recurrir a técnicas complejas como son los injertos óseos o la regeneración tisular guiada ${ }^{3-4}$.

La posibilidad de insertar implantes en crestas óseas estrechas, de una forma poco traumática, reduciendo mínimamente el fresado, ha sido posible gracias a la introducción de un instrumental adecuado como son los expansores u osteodilatadores. Este instrumental modificado por los distintos autores se ha utilizado también para la inserción de implantes con la elevación sinusal y en la pterigoides ${ }^{5}$. Esta técnica relativamente no invasiva consigue tasas de éxito y supervivencia de los implantes similares a las técnicas convencionales de inserción de implantes ${ }^{6}$.

En líneas generales, el aumento del reborde alveolar mediante una corticotomía y posterior expansión de la cresta para la inserción de los implantes ha sido sugerida por algunos autores para evitar el trauma de la realización de injertos y la exposición de membranas, aunque representa una técnica que requiere experiencia quirúrgica suficiente por el profesional ${ }^{7-8}$.

Dos estudios españoles valoran las indicaciones y los resultados del uso de expansores en implantología oral 9-10. El primero compara los resultados clínicos de la inserción de los implantes según la técnica convencional (supervivencia del $98 \%$ hasta la carga funcional) y la realizada con osteodilatadores (supervivencia del $93 \%$ hasta la carga funcional) sin diferencias a los 2,7 años con una tasa de éxito del $90 \%$ para los implantes insertados con osteodilatadores y 92 insertados de forma convencional. Teniendo en cuenta que los casos tratados con expansores presentaban una menor cantidad horizontal de hueso alveolar ${ }^{9}$.

El segundo estudio español confirma estos hallazgos clínicos con un $100 \%$ de éxito a los nuevos meses utili- zando expansores en 26 pacientes para insertar 74 implantes en el área posterior del maxilar superior consiguiendo un aumento de la cresta ósea de 4,5 a 8 mm de anchura ${ }^{10}$.

Un estudio italiano reciente realizado en 45 pacientes con reabsorción horizontal de las crestas alveolares, demuestra que la corticotomía y posterior expansión ósea puede obtener suficiente base ósea para insertar con éxito los implantes. 110 implantes fueron insertados según este método con un éxito del $97,8 \%$ después de un seguimiento medio de 20,4 meses. Los 3 fracasos fueron previos a la restauración prostodóncica ${ }^{11}$.

El objetivo del presente estudio era la valoración clínica y el seguimiento del tratamiento con implantes oseointegrados insertados mediante la técnica de expansión ósea.

\section{PACIENTES Y MÉTODOS}

El presente estudio fue realizado por profesores de las unidades docentes de Odontología Integrada de Adultos de la Facultad de Odontología y del Postgrado de Implantología Oral de la Universidad de Sevilla y del Departamento de Cirugía Oral de la Facultad de Estomatología de La Habana (Cuba).

Previamente a la realización del estudio, aquellos pacientes que padecían trastornos sistémicos graves que podían comprometer la oseointegración fueron excluidos del estudio ${ }^{12}$. Los pacientes seleccionados eran adultos, de ambos sexos.

Todos los pacientes seleccionados en el estudio fueron informados de la técnica quirúrgica de inserción de implantes mediante la expansión ósea así como del protocolo posterior del tratamiento incluyendo los aspectos prostodóncicos, temporalización y seguimiento, y de la posibilidad de la existencia de complicaciones y pérdida de implantes.

Los pacientes autorizaron el tratamiento implantológico mediante un consentimiento informado. Antes del tratamiento, todos los pacientes fueron evaluados radiológicamente, con una ortopantomografía.

Los criterios de éxito y supervivencia de los implantes fueron los recomendados por van Steenberghe et al ${ }^{13}$. En este sentido, la supervivencia fue definida como la proporción de implantes permanentes en su locali- 
zación original aunque no tengan valor clínico o cause efectos adversos. Los criterios de éxito de los implantes se expresan en la Tabla 1.

\section{Cirugía}

Una hora antes de la cirugía, los pacientes comenzaron un régimen antibiótico preventivo (amoxicilina + clavulánico) durante una semana. En casos de molestias, dolor o inflamación a todos los pacientes se les recomendó ibuprofeno. Todos los pacientes recibieron anestesia local. A todos los pacientes se les recomendó el enjuague diario con clorhexidina durante los primeros 30 días.

Se realizó la técnica de expansión ósea que esencialmente consiste en la realización de una corticotomía con un disco o una fresa inicial o mediante un bisturí ultrasónico Piezosurgery ${ }^{\circledR}$ (Mectron, Génova, Italia) y la inserción de los expansores de forma progresiva incrementando su diámetro hasta la inserción del implante correspondiente.

Tanto los expansores utilizados como los implantes de conexión externa insertados (roscados de superficie con chorreado de arena y grabado ácido) fueron Microdent ${ }^{\circledR}$ (Microdent, Barcelona, España) (Figura 1).

\section{TABLA 1.- LOS CRITERIOS DE EXITO EN EL TRATAMIENTO CON IMIPLANTES (van Steenberghe et al ${ }^{13}$ )}

1. El implante no provoca ninguna reacción alérgica, tóxica o infecciosa de carácter local o sistémico.

2. El implante ofrece soporte para una prótesis funcional.

3. El implante no muestra signos de fractura o incurvación.

4. El implante no muestra ninguna movilidad cuando es explorado manual o electrónicamente.

5. El implante no muestra ningún signo de radiolucidez mediante una radiografía intraoral.

6. La pérdida marginal de hueso ( $R x$ intraoral) y/o la pérdida de inserción (profundidad de sondaje + recesión) no deben perjudicar la función de anclaje del implante o causar molestias para el paciente durante 20 años.

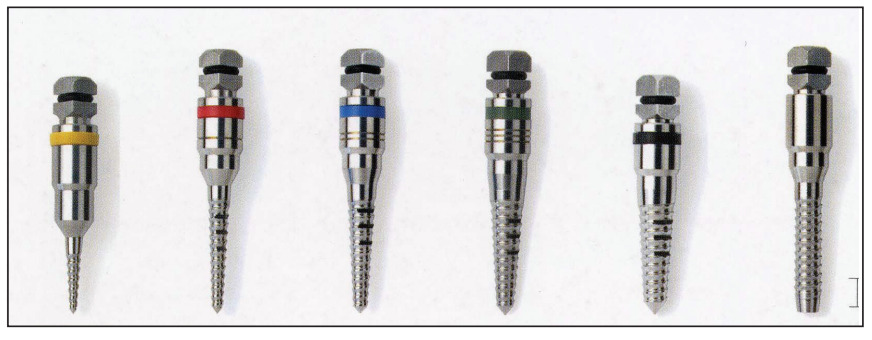

Fig. 1: Kit de expansores roscados Microdent ${ }^{\circledR}$.

\section{Prostodoncia}

A los 3-6 meses después de la inserción de los implantes (dependiendo de la mandíbula o el maxilar superior), se realizó la carga funcional de los implantes mediante la colocación de las correspondientes prótesis implantosoportadas. El tiempo transcurrido de seguimiento clínico desde la carga funcional de los implantes fue al menos de 12 meses.

\section{Análisis estadístico}

Se realizó una estadística descriptiva de los hallazgos clínicos del estudio, con referencia a las variables demográficas de los pacientes, los implantes y prótesis realizadas.

\section{RESULTADOS}

\section{Pacientes}

56 pacientes ( 22 hombres y 34 mujeres) fueron tratados con implantes insertados mediante la técnica de expansión ósea, con una edad media de 39,6 años (rango: 18-72 años).

\section{Implantes}

Se insertaron un total de 117 implantes Microdent ${ }^{\circledR}$ en los correspondientes 56 pacientes, lo que representa una media de 2,1 implantes por paciente. Todos los implantes utilizados, eran de diámetro universal o estándar (3,8-4,2 mm). Con respecto a la longitud, fueron insertados 3 implantes de $14 \mathrm{~mm}, 111$ de $12 \mathrm{~mm}$, y 3 de $10 \mathrm{~mm}$.

106 implantes $(90,5 \%)$ fueron insertados en el maxilar superior mientras que 11 implantes $(9,5 \%)$ en la mandíbula. De todos los implantes insertados, 61 implan- 
tes fueron insertados en el sector anterior $(52,1 \%)$ (incisivos y caninos) de ambos maxilares (50 en el maxilar superior y 11 en la mandíbula); mientras que 56 implantes $(47,9 \%)$ fueron insertados en el sector posterior (exclusivamente maxilar superior).

En 2 implantes $(1,7 \%)$ hubo complicaciones, al presentar movilidad a la exploración clínica durante la fase de cicatrización libre de carga funcional y fueron extraídos. Ningún implante se ha perdido después de la carga funcional prostodóncica durante el periodo de seguimiento clínico.

\section{Prótesis implantosoportadas}

Los 115 implantes restantes fueron cargados funcionalmente, a los 3-6 meses, con sus correspondientes prótesis implantosoportadas. Se realizaron un total de 61 prótesis $(100 \%), 36$ coronas unitarias $(59,1 \%), 19$ puentes fijos $(31,1 \%), 3$ rehabilitaciones fijas $(4,9 \%)$ y 3 sobredentaduras $(4,9 \%)$. El seguimiento clínico medio ha sido de 14,1 meses (rango: 12-24 meses). No se ha perdido ningún implante en este periodo.

\section{DISCUSIÓN}

Este estudio valora los resultados clínicos del tratamiento con implantes oseointegrados insertados mediante la técnica de expansión ósea. Desde que Summers introdujo su técnica con osteótomos en 1994 se ha ido incorporando a la práctica implantológica con buenos resultados clínicos ${ }^{14}$.

La técnica quirúrgica es relativamente sencilla, aunque si el profesional tiene suficiente experiencia clínica y la expansión ósea se realiza cuidadosamente presenta pocas complicaciones y permite la inserción de los implantes en la misma sesión ${ }^{5}$.

La técnica consiste en la introducción progresiva de los diversos expansores de menor a mayor diámetro, después de la corticotomía y fresado inicial, hasta conseguir la expansión adecuada para la inserción del implante correspondiente. Los expansores Microdent ${ }^{\circledR}$ utilizados en el presente estudio se introducen, de forma progresiva, manualmente, presionando y rotando al mismo tiempo, hasta alcanzar la longitud adecuada.

Cuando se realiza la expansión de la cresta alveolar estrecha para la inserción de varios implantes, es necesario realizar una corticotomía a lo largo del rebor- de alveolar e ir repitiendo el procedimiento básico de expansión por cada implante, teniendo la precaución de dejar insertado el último expansor o el implante correspondiente para que no se colapse el hueso mientras expandimos el resto del reborde e insertamos los restantes implantes (Figuras 2-6) ${ }^{5}$.

Los expansores u osteótomos como instrumental implantológico para incrementar la anchura de la cresta alveolar puede ser también utilizada para la elevación atraumática del seno maxilar, ya que representa una técnica quirúrgica menos invasiva que la apertura lateral mediante una ventana directa al seno (elevación traumática). En este sentido, mejora la aceptación por el paciente ya que el tratamiento se realiza en una se-

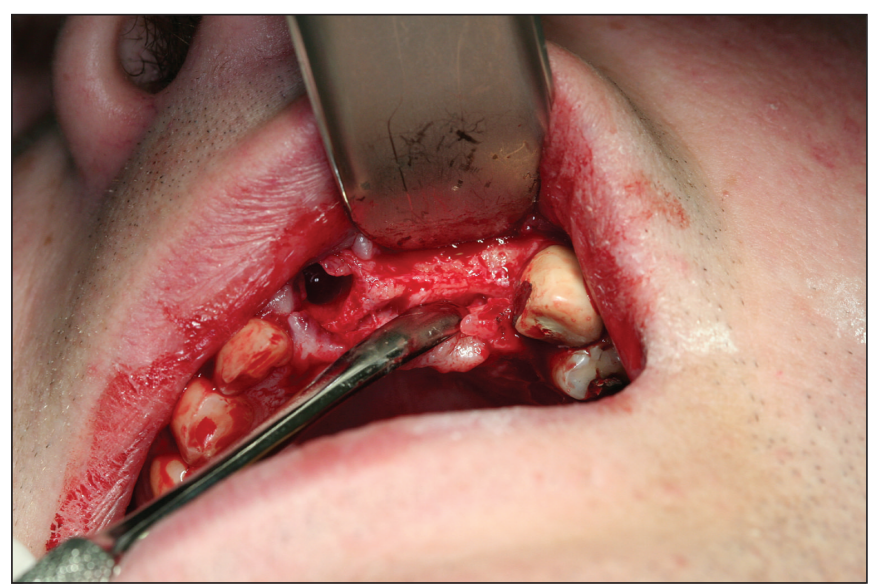

Fig. 2: Aspecto clínico del paciente con pérdida dental en la zona anterior del maxilar superior. Se observa extracción realizada en la misma sesión quirúrgica.

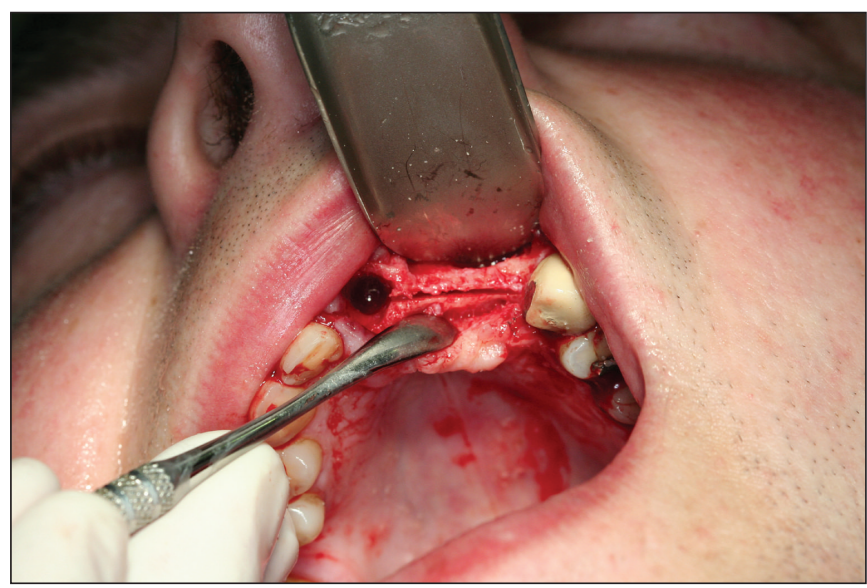

Fig. 3: Aspecto clínico del paciente con corticotomía realizada con bisturí ultrasónico Piezosurgery ${ }^{\circledR}$. 


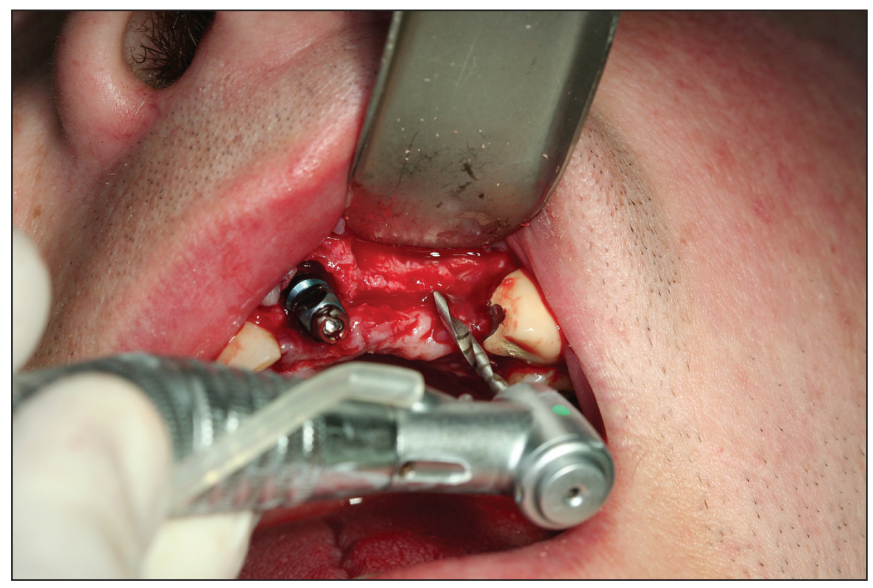

Fig. 4: Inserción inmediata de implante postextracción. Fresado inicial de lechos implantarios.

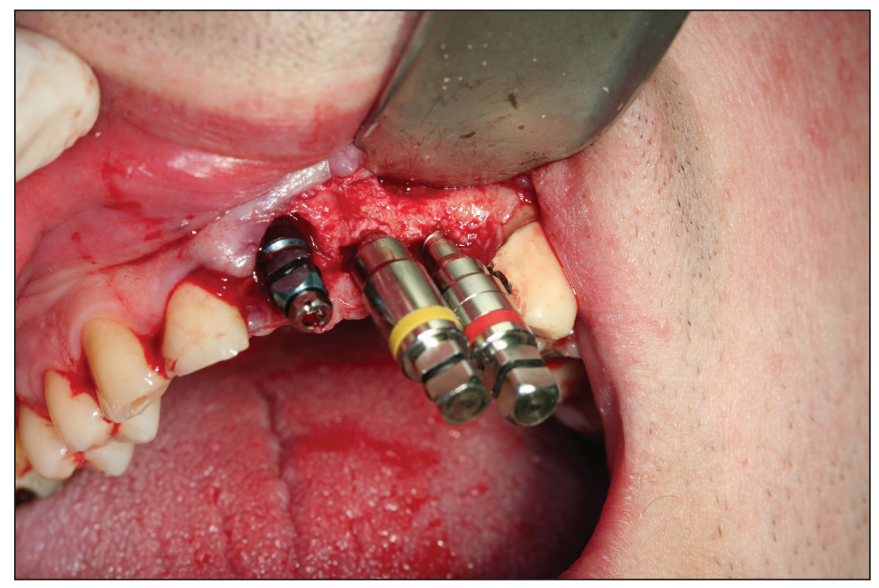

Fig. 5: Inserción y expansión sucesiva de los expansores Microdent ${ }^{\circledR}$.

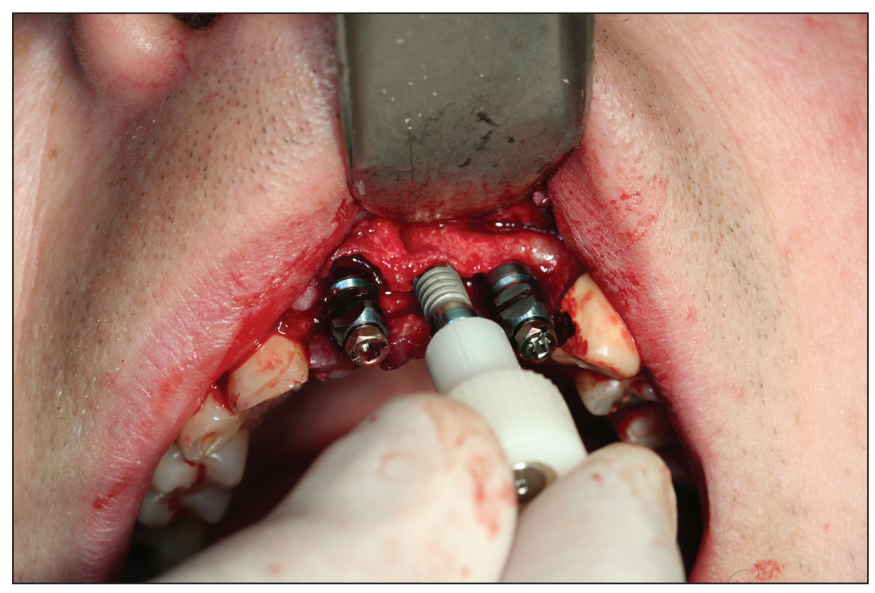

Fig. 6: Inserción definitiva de los implantes. sión quirúrgica comparada con las dos o tres sesiones de la técnica quirúrgica traumática o directa. Un tiempo de cicatrización de 3 meses es suficiente, en comparación con la técnica traumática que necesita un periodo de 6-9 meses ya que además se utiliza biomaterial de relleno ${ }^{15}$.

Los resultados clínicos de la inserción de implantes mediante osteótomos suelen ser similares a los insertados de forma convencional. En este sentido, una reciente metaanálisis sobre 164 estudios iniciales utilizando esta técnica demuestra un éxito entre el $94 \%$ al $100 \%$ después de una media de 56 meses de seguimiento clínico ${ }^{16}$. Estos hallazgos coinciden con los resultados del presente estudio que demuestran una elevada tasa de éxito $(98,3 \%)$ de los implantes insertados con la técnica de expansión ósea para un periodo de seguimiento medio de 14,1 meses.

Se ha investigado en animales de experimentación el grado de oseointegración que se obtiene con los osteótomos, demostrándose que está técnica incrementa, inicialmente de forma significativa, la neoformación ósea y estimula los mecanismos de la oseointegración en el hueso trabecular como expresa un estudio realizado en conejos que valora el contacto hueso-implante mediante histomorfometría y microscopia de fluorescencia ${ }^{17}$. En este sentido, la mejoría en la densidad ósea como consecuencia del uso de expansores favorecería la estabilidad primaria de los implantes insertados con esta técnica, sobre todo en el caso de hueso tipo 4 (zona posterior del maxilar superior). Además, la técnica con expansores presenta menos posibilidades de sobrecalentamiento del lecho óseo en comparación con el fresado clásico ${ }^{17}$.

Sin embargo, otro estudio realizado en minicerdos compara la estabilidad primaria de los implantes que se consigue con la técnica convencional y con la de osteótomos ${ }^{18}$. Mediante un test de remoción por torque se demuestra una menor estabilidad primaria en la técnica con osteótomos después de los 28 días probablemente debido a una insuficiente regeneración ósea. Además, se demuestra que los osteótomos provocan microfracturas trabeculares. Sin embargo no hay diferencias significativas entre ambas técnicas cuando se evalúa la estabilidad primaria con la frecuencia de resonancia ${ }^{18}$.

Los expansores u osteótomos crean un lecho óseo adecuado para la posterior inserción de los implantes dentales según su diámetro y longitud. De esta forma, se desarrolla un aumento horizontal del hueso crestal que proporciona el contorno óseo adecuado para la inser- 
ción del implante ${ }^{19}$. Así mismo, esta cuidadosa técnica manual permite, aprovechando la elasticidad natural del hueso especialmente del maxilar superior, ir configurando progresivamente de acuerdo a la experiencia del profesional una posición tridimensional favorable del implante para su rehabilitación prostodóncica ${ }^{19}$.

La principal complicación del uso de los expansores u osteótomos es la fractura de la cortical ósea vestibular. Este acontecimiento quirúrgico adverso puede ocurrir durante la preparación del lecho mediante el fresado inicial o la utilización de los expansores óseos o durante la inserción del implante ${ }^{19}$. Para evitar estas complicaciones se han ido perfeccionando los diversos sets o kits de osteótomos o expansores de impactados (ej. osteótomos de Summers) a roscados (expansores Microdent ${ }^{\circledR}$ ) que proporcionan una cuidadosa preparación del lecho implantario.

En el maxilar superior posterior es más fácil insertar los expansores que van condensando el hueso y el mayor problema surge para conseguir una buena estabilidad primaria de los implantes ${ }^{11}$. De hecho el 90,5\% de los implantes del presente estudio han sido insertados en el maxilar superior. En este sentido, los implantes actuales configurados con un diseño macroscópico roscado muy favorable proporcionan una mayor estabilidad primaria y su superficie rugosa estimula notablemente la respuesta ósea ${ }^{20}$. Además, se han desarrollado nuevos implantes para conseguir una mejor adaptación al lecho implantario conseguido con los expansores u osteótomos (ej. los implantes de núcleo expansivo MV Microdent ${ }^{\circledR}$ o ITI TE ${ }^{\circledR}$ ).

El presente estudio muestra también los resultados de una menor proporción $(9,5 \%)$ de implantes insertados en la zona anterior mandibular con los expansores. En estos casos, existe un mayor riesgo de fractura porque el módulo de elasticidad de la mandíbula es menor de acuerdo a su estructura anatómica e histológica que proporciona un hueso más duro que en el maxilar superior ${ }^{11,21}$.

Recientemente han aparecido estudios que realizan la inserción de los implantes en la zona posterior mandibular en 2 fases quirúrgicas ${ }^{21}$. En la primera realizan la corticotomía crestal con ampliaciones verticales por mesial y distal con instrumental piezoeléctrico y en una segunda fase (a los 40 días) realizan la expansión ósea y la inserción de los implantes. De esta forma se acorta el tiempo de realizar injertos y se previene la aparición de fracturas debidas a la dureza de la zona mandibular posterior ${ }^{21}$.

\section{CONCLUSIONES}

El desarrollo de la implantología oral actual ha hecho posible que el tratamiento con implantes dentales pueda ser una realidad aún en los casos de poca disponibilidad ósea. En este sentido, además de los injertos óseos, otras alternativas terapéuticas como son la utilización de osteótomos o expansores óseos pueden contribuir a solucionar con éxito estos casos implantológicos más complejos de crestas alveolares más estrechas.

\section{ABSTRACT}

Introduction. The aim of this study was to report the outcome of treatment with dental implants inserted by bone expansion technique.

Methods. 56 patients with tooth loss were treated with 117 Microdent ${ }^{\circledR}$ sandblasted surface implants for rehabilitation by bone expansion technique with Microdent ${ }^{\circledR}$ screw bone expanders. Implants were loaded after a healing free-loading period of 3-6 months according insertion in mandible or maxilla.

Results. Clinical results indicate a survival and success rate of implants of $98,3 \% .2$ implants were lost during the healing period. $90,5 \%$ of implants were inserted in maxilla and $9.5 \%$ in mandible. After a mean functioning period of 14,1 months, no late complications were reported. $59.1 \%$ of patients were restored with single crowns; $31.1 \%$ with fixed bridges; $4.9 \%$ with fixed rehabilitations and $4.9 \%$ with overdentures.

Conclusions. This study indicate that treatment with dental implants by bone expansion constitute a alternative successful implant treatment.

\section{KEY WORDS}

Dental implants, bone expansion, osteotomes, bone expanders, implant dentistry.

\section{BIBLIOGRAFÍA}

1. Albrektsson T, Wennerberg A. The impact of oral implants. Past and future, 1966-2042. J Can Dent Assoc 2005; 71: 327-327d.

2. Misch CE. Contemporary Implant Dentistry. $2^{\mathrm{a}}$ ed. San Luís: Mosby. 1999. 
3. Hammerle CH, Jung RF, Feloutzis A. A systematic review of the survival of implants in bone sites augmented with barrier membranes (guided bone regeneration) in partially edentulous patients. J Clin Periodontol 2002; 29: 226-31.

4. Pérez O, Velasco E, González L, García A, Rodríguez O. Técnicas quirúrgicas complejas en el tratamiento con implantes oseointegrados del maxilar superior. Av Perio Impl Oral 2006; 18: 10-9.

5. Rambla J, Peñarrocha M, Guarinos J. Análisis del uso de los osteodilatadores para la creación del lecho implantológico. Aportaciones técnicas y revisión de la literatura. Med Oral Patol Oral Cir Bucal 2006; 11 : 173-7.

6. Chiapasco M, Zaniboni M, Boisco M. Augmentation procedures for the rehabilitation of deficient edentulous ridges with oral implants. Clin Oral Impl Res 2006; 17 (Suppl. 2): 136-59.

7. Scipioni A, Bruschi GB, Calesini G. The edentulous ridge expansion technique: a five year study. In $\mathrm{t} J$ Perio Rest Dent 1994; 14: 451-9.

8. Sethi A, Kaus T. Maxillary ridge expansion with simultaneous implant placement: 5-year results of an ongoing clinical study. Int J Oral Maxillofac Implants 2000; 15: 491-499.

9. Peñarrocha M, Sanchís JM, Guarinos J, Soriano I, Balaguer J. Estudio comparativo entre la técnica convencional y la de los osteodilatadores para la creación del lecho implantológico. A propósito de 226 implantes colocados en 80 pacientes. Periodoncia 2000; 10: 189-98.

10. Calvo JL, Sáez R, Pardo G, Carión MJ. Ensanchamiento del reborde alveolar posterior del maxilar superior con osteotomos compresivos. Estudio prospectivo a 9 meses. Rev Int Prot Estomatol 2005; 7: 272-8.

11. Chiapasco M, Ferrini F, Casentini P, Accardi S, Zaniboni M. Dental implants placed in expanded narrow edentulous ridges with the Extension Crest device. A 1-3 year multicenter follow-up study. Clin Oral Impl Res 2006; 17: 265-72.

12. Buser D, von Arx T, ten Bruggenkate C, Weingart D. Basic surgical principles with ITI implants. Clin Oral Impl Res 2000; 11 (suppl 1):59-68.

13. van Steenberghe D, Quirynen, Naert I. Survival and success rates with oral endosseous implants. En: Lang
NP, Karring T, Lindhe J (eds.). Proceedings of the 3rd European Workshop on Periodontology. Implant. Dentistry. Berlin: Quintessence. 1999. pag: 242-52.

14. Summers RB. A new concept in maxillary implant surgery: the osteotome technique. Compendium 1994; 15:152-8.

15. Nedir R, Bischof M, Vazquez L, Szmukler-Moncler S, Bernard JP. Osteotome sinus floor elevation without grafting material: a l-year porspective pilot study with ITI implants. Clin Oral Impl Res 2006; 17: 679-86.

16. Shalabi MM, Manders P, Mulder J, Jansen JA, Creughers A. A meta-analysis of clinical studies to estimate the 4.5year survival rate of implants placed osteotome technique. Int J Oral Maxillofac Implants 2007;22: 110-6.

17. Nkenke E, Kloss F, Wiltfang J, Schultze-Mosgau S, Radespiel-Tröger M, Loos K, Neukan FW. Histomorphometric and fluorescence microscopic analysis of bone remodelling after instalaltion of implants using an osteotome technique. Clin Oral Impl Res 2002; 13: 595-602.

18. Bütcher A, Kleinheinz J, Wiesmann HP, Kersken J, Nienkemper M, von Weyhrother H, Joos U, Meyer U. Biological and mechanical evaluation of bone remodelling and implant stability after using an osteotome technique. Clin Oral Impl Res 2005; 16: 1-8.

19. Ferrigno N, Laureti M. Surgical advantages with ITI TE ${ }^{\circledR}$ implants placement in conjunction with split crest technique. 18-month results of an ongoing prospective study. Clin Oral Impl Res 2005; 16: 147-55.

20. Molly L. Bone density and primary stability in implant therapy. Clin Oral Impl Res 2006; 17 (suppl. 2): 124-35.

21. Enislidis G,Wittwer G, Ewers R. Preliminary report on a staged ridge splitting technique for implant placement in the mandible: a technical note. Int J Oral Maxillofac Implants 2006;21:445-9.

\section{CORRESPONDENCIA}

Prof. Eugenio Velasco Ortega

Facultad de Odontología de Sevilla.

$\mathrm{C} /$ Avicena $\mathrm{s} / \mathrm{n}$

41009 Sevilla

Tfno: 954481132

e-mail:evelasco@us.es 